\title{
ON STEADY STATES OF VAN DER WAALS FORCE DRIVEN THIN FILM EQUATIONS
}

\author{
HUIQIANG JIANG AND WEI-MING NI
}

\begin{abstract}
Let $\Omega \subset \mathbb{R}^{N}, N \geq 2$ be a bounded smooth domain and $\alpha>1$. We are interested in the singular elliptic equation

$$
\triangle h=\frac{1}{\alpha} h^{-\alpha}-p \quad \text { in } \Omega
$$

with Neumann boundary conditions. In this paper, we gave a complete description of all continuous radially symmetric solutions. In particular, we constructed nontrivial smooth solutions as well as rupture solutions. Here a continuous solution is said to be a rupture solution if its zero set is nonempty. When $N=2$ and $\alpha=3$, the equation has been used to model steady states of van der Waals force driven thin films of viscous fluids. We also considered the physical problem when total volume of the fluid is prescribed.
\end{abstract}

\section{INTRODUCTION}

The equation

$$
h_{t}=\nabla \cdot\left(h^{3} \nabla p\right)
$$

has been used to model the dynamics of van der Waals force driven thin films of viscous fluids 27 28] 29]. Here $h$ is the thickness of the thin film and the pressure

$$
p=\frac{1}{3} h^{-3}-\triangle h,
$$

is a sum of contributions from disjoining pressure due to attractive van der Waals force and a linearized curvature term corresponding to surface tension effects. Hence, (1.1) becomes

$$
h_{t}=-\nabla \cdot\left(h^{-1} \nabla h\right)-\nabla \cdot\left(h^{3} \nabla \triangle h\right),
$$

which is a special case of the generalized thin film equation

$$
h_{t}=-\nabla \cdot\left(h^{m} \nabla h\right)-\nabla \cdot\left(h^{n} \nabla \triangle h\right)
$$

where the exponents $m, n$ represent the powers in the destabilizing second-order and the stabilizing fourth-order diffusive terms, respectively. This class of equations occurs in connection with many physical models involving fluid interfaces 23] 24]. For example, when $n=1$ and $m=1$, it describes a gravity driven Hele-Shaw cell 11 911 14 25]; for $n=m=3$ it describes fluid droplets hanging from a ceiling [15]; and for $n=0$ and $m=1$, it is a modified Kuramoto-Sivashinsky equation which describes solidification of a hyper-cooled melt [4] [7. Over the past two

Date: August 1, 2018.

2000 Mathematics Subject Classification. Primary 35J25,76A20; Secondary 34B18.

Key words and phrases. Singular elliptic equation, van der Waals, Thin film, Rupture. 
decades, these models have also been the focus of rigorous and extensive mathematical analysis 20 3] 6] 8] 10] 12] [16] 20] 21] 22] 26].

As in the van der Waals force case, when $n-m \neq 1$, letting

$$
p=-\frac{1}{m-n+1} h^{m-n+1}-\triangle h,
$$

we can rewrite (1.4) as

$$
h_{t}=\nabla \cdot\left(h^{n} \nabla p\right) .
$$

Now we consider viscous fluids in a cylindrical container whose bottom is represented by $\Omega$, a bounded smooth domain in $\mathbb{R}^{2}$. Since there is no flux across the boundary, we have the Neumann boundary condition

$$
\frac{\partial p}{\partial \nu}=0 \text { on } \partial \Omega \text {. }
$$

We also ignore the wetting or nonwetting effect, and assume that the fluid surface is perpendicular to the boundary of the container, i.e.,

$$
\frac{\partial h}{\partial \nu}=0 \text { on } \partial \Omega \text {. }
$$

Whenever $m-n \neq-1$ or -2 , we can associate (1.4) with energy

$$
E(h)=\int_{\Omega}\left(\frac{1}{2}|\nabla h|^{2}-\frac{1}{(m-n+1)(m-n+2)} h^{m-n+2}\right),
$$

and formally, using (1.6), (1.7), we have

$$
\begin{aligned}
& \frac{d}{d t} E(h)=\int_{\Omega}\left(-\triangle h h_{t}-\frac{1}{m-n+1} h^{m-n+1} h_{t}\right) \\
= & \int_{\Omega} p \nabla \cdot\left(h^{n} \nabla p\right)=-\int_{\Omega} h^{n}|\nabla p|^{2} .
\end{aligned}
$$

Hence, for a thin film fluid at rest, $p$ has to be a constant, and $h$ satisfies (1.5).

Therefore, letting $\alpha=-(m-n+1)$, we are led to the elliptic problem

$$
\left\{\begin{array}{lll}
\triangle h=\frac{1}{\alpha} h^{-\alpha}-p & \text { in } & \Omega, \\
\frac{\partial h}{\partial \nu}=0 & \text { on } & \partial \Omega,
\end{array}\right.
$$

where $\Omega$ is a bounded smooth domain in $\mathbb{R}^{N}, N \geq 1$ and $p$ is a constant.

When $N=1$, this equation has been studied by R. Laugesen and M. Pugh in 20] where they produced positive, smooth steady states for all $\alpha$ and touchdown steady states for $\alpha<1$. In [5], A. L. Bertozzi, G. Grün and T. P. Witelski considered (1.8) with additional Born repulsion term which leads to the elliptic equation

$$
\begin{cases}\triangle h=\frac{1}{\alpha} h^{-\alpha}\left(1-\left(\frac{\varepsilon}{h}\right)^{\beta}\right)-p & \text { in } \Omega, \\ \frac{\partial h}{\partial \nu}=0 & \text { on } \partial \Omega,\end{cases}
$$

where $\beta$ is a positive constant. When $\varepsilon>0$, the associated energy to (1.9) is bounded from below which makes a variational approach possible and enables them to show the existence of an energy minimizer in any dimensions. It seems difficult to extend this approach to the limiting case $\varepsilon=0$.

The goal of this paper is to understand radial solutions of (1.8) when $N \geq 2$ and $\alpha>1$ which we will assume throughout this paper. In particular, when $N=2$ and $\alpha=3$, we come to the van der Waals force driven thin films in the physically realistic dimension. When $\alpha>1$, except the limited discussions in [18, there 
seems no established elliptic theory for (1.8), and hence it is the mathematically more interesting case. We remark that energy method can be applied to yield nontrivial solutions to (1.8) when $\alpha<1$. On the other hand, the behavior of radial solutions is also quite different when $\alpha>1$. For example, we will show that for $N \geq 2$, the radial solutions will never vanish away from the origin which contrasts with the $\alpha<1$ case where touchdown steady states can be shown to exist in any dimensions.

Due to the singular nature of (1.8), we need to be careful in discussing "solutions" to (1.8). We say $h$ is a continuous solution of (1.8) in $\Omega$, if $h \neq \equiv 0$ and is a nonnegative continuous function in $\bar{\Omega}$ satisfying the equation in (1.8) in the open set $\{x \in \Omega: h(x)>0\}$. The rupture set of $h$,

$$
\Sigma=\{x \in \Omega: h(x)=0\},
$$

corresponds to "dry spots" in the thin film, which is of great significance in the coatings industry where nonuniformities are very undesirable. Standard elliptic theory implies that $h$ is smooth and hence a classical solution of (1.8) in $\Omega \backslash \Sigma$. An interesting Hausdorff dimension estimate of $\Sigma$ can be found in 18 where it is shown that any finite energy solution satisfies $\mathcal{H}^{\mu}(\Sigma)=0$ where $\mu=N-2+\frac{4}{\alpha+1}$. For van der Waals force driven thin film, we have $N=2$ and $\alpha=3$, hence $\mathcal{H}^{1}(\Sigma)=0$, i.e., the thin film with finite energy can't have one dimensional rupture set.

For any $p>0$, let

$$
\xi=(\alpha p)^{-\frac{1}{\alpha}}
$$

then $h \equiv \xi$ is always a solution to (1.6). The natural question is whether it is the only solution. For the radially symmetric case, after a simple scaling, the uniqueness theorem in [13] implies:

Proposition 1.1. Let $N \geq 2$ and $\alpha>1$. For any given $R>0$, there exists a constant $p_{0}$, such that for any $p \leq p_{0}, h \equiv \xi$ is the only radial solution of (1.8) in $B_{R}(0)$.

When $p$ is large, nontrivial solutions do exist. In fact, we have

Theorem 1.2. Let $N \geq 2$ and $\alpha>1$. For any given $R>0$, there exists a nondecreasing sequence of $\left\{p_{k}\right\}$, such that for any $p>p_{k}$, (1.8) has at least $k$ nontrivial smooth radial solutions in $B_{R}(0)$.

Theorem 1.2 is an application of Theorem 1.3 below which gives a complete description of all nontrivial smooth radial solutions.

Theorem 1.3. Let $N \geq 2$ and $\alpha>1$. For any given $p>0$, and for any $\eta>0$, $\eta \neq \xi$, there exists an increasing sequence $\left\{r_{k}^{p, \eta}\right\}_{k=1}^{\infty}$ with

$$
r_{1}^{p, \eta} \geq \max \left\{\sqrt{\frac{2 N \alpha(\eta-\xi)}{\xi^{-\alpha}-\eta^{-\alpha}}},\left(\frac{p_{0}}{p}\right)^{\frac{1+\alpha}{2 \alpha}}\right\}
$$

where $p_{0}$ is the constant in Proposition 1.1, and

$$
\lim _{k \rightarrow \infty}\left(r_{k+1}^{p, \eta}-r_{k}^{p, \eta}\right)=\pi(\alpha p)^{-\frac{1+\alpha}{2 \alpha}},
$$

such that for each $r_{k}^{p, \eta}$, there exists a unique smooth radial solution of (1.8) in $B_{r_{k}^{p, \eta}}(0)$ satisfying $h(0)=\eta$. 
We say a continuous solution to (1.8) is a rupture solution if $\Sigma$ is not empty. It will be shown that for radial solutions, rupture can only occur at the origin. (See Corollary 2.2 below.) Our main result in this paper is as follows.

Theorem 1.4. Let $N \geq 2$ and $\alpha>1$. For any given $p>0$, there exists an increasing sequence $\left\{r_{k}^{p, 0}\right\}_{k=1}^{\infty}$ with

$$
r_{1}^{p, 0} \geq\left(\frac{p_{0}}{p}\right)^{\frac{1+\alpha}{2 \alpha}}
$$

and

$$
\lim _{k \rightarrow \infty}\left(r_{k+1}^{p, 0}-r_{k}^{p, 0}\right)=\pi(\alpha p)^{-\frac{1+\alpha}{2 \alpha}},
$$

where $p_{0}$ is the constant in Proposition 1.1, such that for each $r_{k}^{p, 0}$, there exists a unique radial rupture solution to (1.8) in $B_{r_{k}^{p, 0}}(0)$. Furthermore, if $R \neq r_{k}^{p, 0}$ for any $k$, then there is no radial rupture solution to (1.8) in $B_{R}(0)$.

Remark 1.5. The rupture solutions constructed in Theorem 1.4 above are weak solutions to (1.8) in the distributional sense. (See Remark 4.3 below.) We also remark that when $N=1$ and $\alpha>1$, there is no radial rupture solutions [20].

For any given $p>0$, there exists a nontrivial radial solutions to (1.8) in $B_{R}(0)$ if and only if $r_{k}^{p, \eta}=R$ holds for some $\eta \geq 0, \eta \neq \xi$ and for some integer $k \geq 1$.

In physical experiments, usually the total volume of the fluid is known, i.e., the average film thickness

$$
\bar{h}=\frac{1}{|\Omega|} \int_{\Omega} h(x) d x
$$

is given while the pressure $p$ is an unknown constant. Hence, given $\bar{h}>0$, we need to find function $h$ and constant $p$, such that

$$
\begin{cases}\triangle h=\frac{1}{\alpha} h^{-\alpha}-p & \text { in } \quad \Omega, \\ \frac{1}{\Omega} \int_{\Omega} h(x) d x=\bar{h} & \\ \frac{\partial h}{\partial \nu}=0 & \text { on } \quad \partial \Omega .\end{cases}
$$

When $\Omega=B_{1}(0)$, all radial solutions of (1.14) can be obtained by scaling from solutions in Theorems [1.3 and 1.4 We will discuss such scaling in Section [5] In particular, we will show

Theorem 1.6. Let $N \geq 2, \alpha>1$ and $\Omega=B_{1}(0) \subset \mathbb{R}^{N}$. There exists a sequence of thickness $\bar{h}_{1}, \bar{h}_{2}, \cdots$ satisfying

$$
\lim _{k \rightarrow \infty} \sqrt{k \pi} \bar{h}_{k}=1
$$

such that for any $k$, (1.14) with $\bar{h}=\bar{h}_{k}$ has a radial rupture solution which, viewed as a function in $r$, has exactly $k-1$ critical points in $(0,1)$. Furthermore, if $\bar{h} \neq \bar{h}_{k}$ for any $k$, then (1.14) has no radial rupture solution.

When $\Omega=B_{1}(0)$, Proposition 1.1 implies that nontrivial solutions to (1.14) must satisfy $p>p_{0}$. Since

$$
p=\frac{1}{|\Omega|} \int_{\Omega} \frac{1}{\alpha} h^{-\alpha},
$$

we may ask the existence of a critical average film thickness $\bar{h}_{0}$ so that there is no nontrivial solutions to (1.14) whenever $h \geq \bar{h}_{0}$. Numerical analysis suggests that 
$\bar{h}_{0}$ does not exist. However, we are unable to provide an analytical proof. Such a proof could be possible if we have better understanding of $\bar{h}(p, \eta, k)$ which is defined in section 5. Moreover, the detailed property of $\bar{h}(p, \eta, k)$ could also provide us a statement similar to Theorem 1.6 for the smooth radial solutions.

The paper is organized in the following way: In Section 2, we show that any radial solution can be extended to a global solution which is oscillating around $\xi$. In Section 3 and Section 4, we discuss smooth radial solutions and rupture solutions respectively and Theorems 1.3 and 1.4 are proved. In Section 5, we use scaling argument to prove Theorems 1.2 and 1.6

\section{Preliminaries}

Recall that given $\alpha>1$ and $p>0, h \in C^{0}\left(B_{R}(0)\right)$ is said to be a continuous solution of

$$
\triangle h=\frac{1}{\alpha} h^{-\alpha}-p
$$

in $B_{R}(0)$ if $h \geq 0$ and it satisfies (2.1) in the open set $\left\{x \in B_{R}(0): h(x)>0\right\}$.

Let $h$ be a radially symmetric solution to (2.1), we can view $h$ as a continuous function defined on $[0, R)$ satisfying

$$
h^{\prime \prime}+\frac{N-1}{r} h^{\prime}+f(h)=0
$$

in the set $S^{+}=\{r \in(0, R): h(r)>0\}$. Here

$$
f(h)=-\frac{1}{\alpha} h^{-\alpha}+p
$$

is monotone increasing and its antiderivative

$$
F(h)=\frac{1}{\alpha(\alpha-1)} h^{1-\alpha}+p h
$$

is convex in $(0, \infty)$. Let

$$
\xi=(\alpha p)^{-\frac{1}{\alpha}},
$$

then $F^{\prime}(\xi)=f(\xi)=0$, and $F$ achieves its absolute minimum at $\xi$. Furthermore, $F(h) \rightarrow \infty$ as $h \rightarrow 0^{+}$or $h \rightarrow \infty$.

For each $r \in S^{+}$, letting

$$
\begin{aligned}
& e_{1}(r)=\frac{1}{2}\left(h^{\prime}(r)\right)^{2}+F(h(r)), \\
& e_{2}(r)=\frac{1}{2}\left(r^{N-1} h^{\prime}(r)\right)^{2}+r^{2(N-1)} F(h(r))=r^{2(N-1)} e_{1}(r),
\end{aligned}
$$

we have

$$
\frac{d}{d r}\left[e_{1}(r)\right]=-\frac{N-1}{r}\left(h^{\prime}(r)\right)^{2} \leq 0,
$$

and

$$
\frac{d}{d r}\left[e_{2}(r)\right]=2(N-1) F(h(r)) r^{2 N-3} \geq 0 .
$$

The monotonicity of $e_{1}$ and $e_{2}$ will be used to obtain a priori bounds. 
Lemma 2.1. Let $0<r_{1}<r_{2}$ be such that $\left(r_{1}, r_{2}\right) \subset S^{+}$. Given $\bar{r} \in\left(r_{1}, r_{2}\right)$, we have, for any $r \in\left(r_{1}, r_{2}\right)$,

$$
e_{1}(r) \leq\left(\frac{\bar{r}}{r}\right)^{2(N-1)} e_{1}(\bar{r})
$$

Furthermore,

$$
c_{1} \leq h(r) \leq c_{2}
$$

where $c_{1}, c_{2}$ are two positive constants depending on $\alpha, p, N, r_{1}, \bar{r}, h(\bar{r})$ and $h^{\prime}(\bar{r})$, and are independent of $r_{2}$.

Proof. Since $e_{2}(r)$ is monotone increasing, we have, for any $r \in\left(r_{1}, \bar{r}\right]$,

$$
e_{1}(r)=r^{2(1-N)} e_{2}(r) \leq r^{2(1-N)} e_{2}(\bar{r})=\left(\frac{\bar{r}}{r}\right)^{2(N-1)} e_{1}(\bar{r}) .
$$

On the other hand, since $e_{1}(r)$ is monotone decreasing, we have, for any $r \in\left[\bar{r}, r_{2}\right)$,

$$
e_{1}(r) \leq e_{1}(\bar{r}) .
$$

Combining (2.7) and (2.8), we obtain (2.5). Now for any $r \in\left(r_{1}, r_{2}\right)$,

$$
F(h(r)) \leq e_{1}(r) \leq\left(\frac{\bar{r}}{r_{1}}\right)^{2(N-1)} e_{1}(\bar{r}),
$$

so (2.6) follows from the fact that $F(h) \rightarrow \infty$ as $h \rightarrow 0^{+}$or $h \rightarrow \infty$.

Corollary 2.2. $h$ can not have rupture away from the origin, i.e., $S^{+}=(0, R)$. Furthermore, $h$ can be uniquely extended to a positive smooth solution of (2.2) in $(0, \infty)$.

Proof. Since $S^{+}$is open, it is a union of open intervals of the form $\left(r_{1}, r_{2}\right)$ with $r_{1}, r_{2} \notin S^{+}$. Given any such interval, if $r_{1}>0$, Lemma 2.1] implies

$$
\liminf _{r \rightarrow r_{1}^{+}} h(r)>0,
$$

and since $h$ is continuous, we conclude $h\left(r_{1}\right)>0$, which contradicts the assumption $r_{1} \notin S^{+}$. Similarly, we can get a contradiction if $r_{2}<R$. Hence, $S^{+}=(0, R)$. Extending $h$ to a maximal interval of existence $\left(0, R^{*}\right)$. If $R^{*}<\infty$, applying Lemma 2.1] again, we have for some positive constants $c_{1}, c_{2}$

$$
c_{1} \leq h(r) \leq c_{2} \text { for any } r \in\left(R / 2, R^{*}\right),
$$

so the solution can be extended beyond $R^{*}$. Hence, $R^{*}=\infty$.

Now, redefining $S^{+}=\{r>0: h(r)>0\}$, we observe that $S^{+}=(0, \infty)$ and Lemma 2.1] still holds. In particular, (2.6) holds for all $r_{1}<r<\infty$. In the remaining part of this section, we shall show that $h$ oscillates around $\xi$ near $r=\infty$.

We will need Sturm's Separation Theorem.

Lemma 2.3. 17] Let $q(t)$ be a real-valued continuous function such that

$$
0<m \leq q(t) \leq M .
$$

Given $t_{2}>t_{1}>0$, if $u=u(t)$ is a nontrivial solution of

$$
u^{\prime \prime}+q(t) u=0
$$


satisfying $u(t)>0$ on $\left(t_{1}, t_{2}\right)$, then

$$
t_{2}-t_{1} \leq \frac{\pi}{\sqrt{m}}
$$

And if in addition $u\left(t_{1}\right)=u\left(t_{2}\right)=0$, then

$$
t_{2}-t_{1} \geq \frac{\pi}{\sqrt{M}}
$$

Lemma 2.4. For any $r_{0}>0$, there exists $r_{1}>r_{0}$ such that $h^{\prime}\left(r_{1}\right)=0$.

Proof. Suppose this is false, then we have either $h^{\prime}(r)>0$ for all $r \in\left(r_{0}, \infty\right)$ or $h^{\prime}(r)<0$ for all $r \in\left(r_{0}, \infty\right)$. Hence, $h$ is strictly monotone increasing or decreasing on $\left(r_{0}, \infty\right)$. From Lemma 2.1 and the observation above, it follows that $h$ is also bounded at $\infty$. So we can assume

$$
\lim _{r \rightarrow \infty} h(r)=\zeta
$$

for some $\zeta>0$. For any $r>r_{0}$, integrating (2.2) from $r_{0}$ to $r$, we obtain

$$
h^{\prime}(r)-h^{\prime}\left(r_{0}\right)+\int_{r_{0}}^{r} \frac{N-1}{s} h^{\prime}(s) d s+\int_{r_{0}}^{r} f(h(s)) d s=0 .
$$

Since

$$
\frac{1}{2}\left(h^{\prime}(r)\right)^{2} \leq e_{1}(r) \leq e_{1}\left(r_{0}\right),
$$

$h^{\prime}(r)$ is bounded in $\left[r_{0}, \infty\right)$. Now, as $h^{\prime}(r)$ does not change sign,

$$
\left|\int_{r_{0}}^{r} \frac{N-1}{s} h^{\prime}(s) d s\right| \leq \frac{N-1}{r_{0}}\left|\int_{r_{0}}^{r} h^{\prime}(s) d s\right|=\frac{N-1}{r_{0}}\left|h(r)-h\left(r_{0}\right)\right|
$$

which is also bounded in $\left[r_{0}, \infty\right)$. Hence identity (2.9) implies that

$$
\int_{r_{0}}^{r} f(h(s)) d s
$$

is bounded in $\left[r_{0}, \infty\right)$. Since $\lim _{r \rightarrow \infty} h(r)=\zeta$, we have

$$
\lim _{r \rightarrow \infty} f(h(r))=f(\zeta)
$$

which must be 0 . Thus $\zeta=\xi$ and

$$
\lim _{r \rightarrow \infty} h(r)=\xi
$$

Now let

$$
v(r)=r^{\frac{N-1}{2}}(h(r)-\xi)
$$

then

$$
v_{r r}+\frac{(N-1)(3-N)}{4 r^{2}} v+r^{\frac{N-1}{2}} f(h)=0 .
$$

Since $f(\xi)=0$, we can rewrite (2.10) as

$$
v_{r r}+B(r) v=0,
$$

where

$$
B(r)=\frac{f(h)-f(\xi)}{h-\xi}+\frac{(N-1)(3-N)}{4 r^{2}} .
$$

Now $\lim _{r \rightarrow \infty} h(r)=\xi$ implies

$$
\lim _{r \rightarrow \infty} B(r)=f^{\prime}(\xi)>0 .
$$


By Lemma 2.3

$$
v(r)=r^{\frac{N-1}{2}}(h(r)-\xi)
$$

will be oscillating around 0 as $r \rightarrow \infty$, which contradicts the assumption that $h(r) \rightarrow \xi$ in a strictly monotonic manner.

Next, we have

Lemma 2.5. Let $h^{\prime}\left(r_{0}\right)=0$ for some $r_{0} \geq 0$.

(i). If $h\left(r_{0}\right)=\xi$, then $h(r) \equiv \xi$.

(ii). If $h\left(r_{0}\right)>\xi$, then there exists $r_{1}>r_{0}$, such that $h^{\prime}(r)<0$ on $\left(r_{0}, r_{1}\right)$, $h^{\prime}\left(r_{1}\right)=0, h\left(r_{1}\right)<\xi$ and $F\left(h\left(r_{1}\right)\right)<F\left(h\left(r_{0}\right)\right)$.

(iii). If $0<h\left(r_{0}\right)<\xi$, then there exists $r_{1}>r_{0}$, such that $h^{\prime}(r)>0$ on $\left(r_{0}, r_{1}\right)$, $h^{\prime}\left(r_{1}\right)=0, h\left(r_{1}\right)>\xi$ and $F\left(h\left(r_{1}\right)\right)<F\left(h\left(r_{0}\right)\right)$.

Proof. (i). This is the standard ODE uniqueness result.

(ii). Since $h\left(r_{0}\right)>\xi$, we have $f\left(h\left(r_{0}\right)\right)>0$. Now

$$
\left(r^{N-1} h^{\prime}\right)^{\prime}=-r^{N-1} f(h),
$$

implies that $r^{N-1} h^{\prime}$ is strictly monotone decreasing in $\left(r_{0}, r_{0}+\delta\right)$ for some $\delta>0$. Hence we have $h^{\prime}(r)<0$ on $\left(r_{0}, r_{0}+\delta\right)$. Applying Lemma 2.4 there exists $r_{1}>r_{0}$, such that $h^{\prime}\left(r_{1}\right)=0$, and we also have $h^{\prime}(r)<0$ on $\left(r_{0}, r_{1}\right)$ if we choose the smallest such $r_{1}$. If $h\left(r_{1}\right)>\xi$, we would have $r^{N-1} h^{\prime}$ is strictly decreasing near $r_{1}$, hence $h^{\prime}\left(r_{1}\right)<0$, which gives a contradiction. And if $h\left(r_{1}\right)=\xi$, then $h \equiv \xi$, which contradicts the hypothesis $h\left(r_{0}\right)>\xi$. Hence we have $h\left(r_{1}\right)<\xi$. Finally, $F\left(h\left(r_{1}\right)\right)<F\left(h\left(r_{0}\right)\right)$ follows from (2.3) .

(iii). Similar to the proof of part (ii).

Let $h$ be a nontrivial global solution of (2.2), starting with $r_{1}>0$ such that $h^{\prime}\left(r_{1}\right)=0$. The existence of $r_{1}$ is guaranteed by Lemma 2.4. Without loss of generality, we assume $h\left(r_{1}\right)<\xi$. For $k=1,2, \cdots$, we define through Lemma 2.5.

$$
\begin{aligned}
& r_{2 k}=\sup \left\{r>r_{2 k-1}: h^{\prime}(s)>0 \text { for all } s \in\left(r_{2 k-1}, r\right)\right\}, \\
& r_{2 k+1}=\sup \left\{r>r_{2 k}: h^{\prime}(s)<0 \text { for all } s \in\left(r_{2 k}, r\right)\right\} .
\end{aligned}
$$

Lemma 2.6.

$$
\lim _{k \rightarrow \infty} r_{k}=\infty
$$

Proof. If it is not true, then we have

$$
\lim _{k \rightarrow \infty} r_{k}=r^{*}
$$

for some $r^{*}>0$. Since $h$ is smooth, we have

$$
h\left(r^{*}\right)=\xi, \quad h^{\prime}\left(r^{*}\right)=0,
$$

hence Lemma 2.5 implies $h \equiv \xi$, which is a contradiction.

Next, we show that the lengths of oscillating intervals $r_{k+1}-r_{k}$ are bounded.

Lemma 2.7. There exists positive constants $C_{1}$ and $C_{2}$ such that

$$
C_{1} \leq\left(r_{k+1}-r_{k}\right) \leq C_{2}
$$

for any $k=1,2,3, \cdots$. 
Proof. Since

$$
\lim _{k \rightarrow \infty} r_{k}=\infty
$$

we only need to prove the lemma when $r_{k}$ is sufficiently large. Differentiating (2.2), we have

Let

$$
h^{\prime \prime \prime}+\frac{N-1}{r} h^{\prime \prime}-\frac{N-1}{r^{2}} h^{\prime}+h^{-\alpha-1} h^{\prime}=0 .
$$

then $w$ satisfies

$$
w(r)=r^{\frac{N-1}{2}} h^{\prime}(r)
$$

Since $h$ is bounded away from both zero and infinity when $r \rightarrow \infty$ by (2.6), we have for some $R>0$ such that for any $r>R$,

$$
c_{1} \leq h^{-\alpha-1}(r)-\frac{N^{2}-1}{4 r^{2}} \leq c_{2}
$$

for some positive constants $c_{1}, c_{2}$. Since $r_{k}, k=1,2, \cdots$, are zeros of $w$, the conclusion follows from Lemma 2.3

Finally, we have

\section{Lemma 2.8.}

$$
\lim _{r \rightarrow \infty} h(r)=\xi .
$$

Proof. Starting with $r_{1}>0$ such that $h^{\prime}\left(r_{1}\right)=0, h\left(r_{1}\right)<\xi$, we define $r_{k}$ as above. Since $F\left(h\left(r_{k}\right)\right)$ is monotone decreasing in $k$, and $h\left(r_{2 k}\right)>\xi, h\left(r_{2 k-1}\right)<\xi$, the property of function $F$ implies $h\left(r_{2 k}\right)$ is monotone decreasing and $h\left(r_{2 k-1}\right)$ is monotone increasing. Hence we have the limits

$$
\eta_{1} \equiv \lim _{k \rightarrow \infty} h\left(r_{2 k}\right) \geq \xi \geq \eta_{2} \equiv \lim _{k \rightarrow \infty} h\left(r_{2 k-1}\right) .
$$

Now

$$
\begin{aligned}
& \eta_{1}-\eta_{2} \leq\left|h\left(r_{k+1}\right)-h\left(r_{k}\right)\right|=\int_{r_{k}}^{r_{k+1}}\left|h^{\prime}(r)\right| d r \\
\leq & \left(r_{k+1}-r_{k}\right)^{\frac{1}{2}}\left(\int_{r_{k}}^{r_{k+1}}\left|h^{\prime}\right|^{2} d r\right)^{\frac{1}{2}} \leq \sqrt{C_{2}}\left(\int_{r_{k}}^{r_{k+1}}\left|h^{\prime}\right|^{2} d r\right)^{\frac{1}{2}},
\end{aligned}
$$

which implies

$$
\int_{r_{k}}^{r_{k+1}} \frac{\left|h^{\prime}\right|^{2}}{r} d r \geq \frac{1}{r_{k+1}} \int_{r_{k}}^{r_{k+1}}\left|h^{\prime}\right|^{2} \geq \frac{\left(\eta_{1}-\eta_{2}\right)^{2}}{C_{2} r_{k+1}} \geq \frac{\left(\eta_{1}-\eta_{2}\right)^{2}}{2 C_{2}^{2}} \int_{r_{k}}^{r_{k+1}} \frac{1}{r} d r
$$

when $k$ is sufficiently large. In the last inequality, we used $r_{k+1} \leq 2 r_{k}$ when $k$ is large. From (2.3), we have for any $r>r_{1}$,

$$
\int_{r_{1}}^{r} \frac{\left|h^{\prime}\right|^{2}}{r}=\frac{1}{N-1}\left[e_{1}\left(r_{1}\right)-e_{1}(r)\right] \leq \frac{e_{1}\left(r_{1}\right)}{N-1} .
$$

Therefore $\frac{\left|h^{\prime}\right|^{2}}{r}$ is integrable at $\infty$. Since $\frac{1}{r}$ is not integrable at $\infty$, (2.13) implies that $\eta_{1}=\eta_{2}=\xi$ and

$$
\lim _{r \rightarrow \infty} h(r)=\xi
$$




\section{Corollary 2.9.}

$$
\lim _{k \rightarrow \infty}\left(r_{k+1}-r_{k}\right)=\pi(\alpha p)^{-\frac{1+\alpha}{2 \alpha}} .
$$

Proof. In equation (2.12), we now have

$$
\lim _{r \rightarrow \infty}\left(h^{-\alpha-1}-\frac{N^{2}-1}{4 r^{2}}\right)=\xi^{-\alpha-1}=(\alpha p)^{\frac{1+\alpha}{\alpha}} .
$$

Hence (2.14) follows from Lemma 2.3 .

\section{Nontrivial Smooth Radially Symmetric Solutions}

This section is devoted to the proof of Theorem 1.3

Given $\eta>0$, we consider (2.2) with the initial values

$$
h(0)=\eta>0, h_{r}(0)=0 .
$$

The local existence and uniqueness of a smooth solution is standard since $f$ is smooth when $h$ is bounded away from zero. And such solution is actually a global solution from Corollary 2.2 For any $\eta \neq \xi$, without loss of generality, we assume $\eta>\xi$. Since

$$
\left(r^{N-1} h^{\prime}\right)^{\prime}=-r^{N-1} f(h)=-r^{N-1}\left(-\frac{1}{\alpha} h^{-\alpha}+p\right),
$$

we have $\left(r^{N-1} h^{\prime}\right)^{\prime}<0$ in $(0, \delta)$ for some small $\delta>0$. From $h^{\prime}(0)=0$, we conclude $h^{\prime}(r)<0$ in $(0, \delta)$. Then we can define

$$
r_{1}=\min \left\{r>0: h^{\prime}(r)=0\right\} .
$$

The existence of $r_{1}>0$ is guaranteed by Lemma 2.4 with $r_{0}=\frac{\delta}{2}$. From the analysis in the previous section, $h$ will be oscillating around $\xi$, and all critical points of $h$ can be listed as $r_{1}<r_{2}<r_{3}<\cdots$, with

$$
C_{1} \leq r_{k+1}-r_{k} \leq C_{2}
$$

and

$$
\lim _{k \rightarrow \infty}\left(r_{k+1}-r_{k}\right)=\pi(\alpha p)^{-\frac{1+\alpha}{2 \alpha}} .
$$

Hence for any $k \geq 1, h$ is a nontrivial smooth solution of

$$
\left\{\begin{array}{lll}
\triangle h=\frac{1}{\alpha} h^{-\alpha}-p & \text { in } & B_{r_{k}}(0) \\
\frac{\partial h}{\partial \nu}=0 & \text { on } & \partial B_{r_{k}}(0)
\end{array} .\right.
$$

And all nontrivial smooth radial solutions of (1.14), when $\Omega$ is a ball, can be obtained this way. More precisely, let $\Omega=B_{R}(0)$ for a given $R>0$, then (1.14) has a nontrivial smooth radial solution if and only if $R=r_{k}^{p, \eta}$ for some $\eta>0, \eta \neq \xi$ and for some $k \geq 1$, here we write $r_{k}=r_{k}^{p, \eta}$ to recognize its dependence on $p$ and $\eta$.

Now we recall the uniqueness result of M. Del Pino and G. Hernandez [13].

Proposition 3.1. Given $\alpha>1$, there exists $d_{0}>0$, such that

$$
\left\{\begin{array}{lll}
-d \triangle u+u^{-\alpha}=1 & \text { in } & B_{1}(0) \\
\frac{\partial u}{\partial \nu}=0 & \text { on } & \partial B_{1}(0)
\end{array}\right.
$$

has no nontrivial radial solution whenever $d \geq d_{0}$. 
It is easy to verify

$$
\tilde{h}(x)=(\alpha p)^{\frac{1}{\alpha}} h\left(r_{1} x\right)
$$

satisfies (3.1) with

$$
d=\frac{\alpha}{(\alpha p)^{\frac{\alpha+1}{\alpha}} r_{1}^{2}} .
$$

Hence Proposition 3.1 implies

$$
r_{1}>\left(\frac{\alpha}{(\alpha p)^{\frac{\alpha+1}{\alpha}} d_{0}}\right)^{\frac{1}{2}} \equiv\left(\frac{p_{0}}{p}\right)^{\frac{\alpha+1}{2 \alpha}},
$$

here

$$
p_{0}=\left(\frac{1}{\alpha^{\frac{1}{\alpha}} d_{0}}\right)^{\frac{\alpha}{\alpha+1}}=\alpha^{-\frac{1}{\alpha+1}} d_{0}^{-\frac{\alpha}{\alpha+1}} .
$$

In general, $r_{k}$ depends on both $p$ and $\eta$. We refer to Corollary 5.2 for the scaling of $r_{k}$ when $p, \eta$ changes.

Lemma 3.2. For any $\eta>0, \eta \neq \xi$, we have

$$
r_{1}(\eta) \geq \sqrt{\frac{2 N \alpha(\eta-\xi)}{\xi^{-\alpha}-\eta^{-\alpha}}} .
$$

In particular,

$$
\lim _{\eta \rightarrow \infty} r_{1}(\eta)=\infty
$$

Proof. First we assume $\eta>\xi$. From the definition of $r_{1}(\eta)$ and Lemma 2.5 we have $h^{\prime}\left(r_{1}\right)=0, h\left(r_{1}\right)<\xi$ and for any $r \in\left(0, r_{1}\right), 0<h(r)<\eta, h^{\prime}(r)<0$. Now

$$
\left(r^{N-1} h^{\prime}\right)^{\prime}=-r^{N-1}\left(\frac{\xi^{-\alpha}}{\alpha}-\frac{h^{-\alpha}}{\alpha}\right) \geq-r^{N-1} \frac{\xi^{-\alpha}-\eta^{-\alpha}}{\alpha} .
$$

Integrating from 0 to $r$, we have

$$
r^{N-1} h^{\prime}(r) \geq-\frac{r^{N}}{N} \frac{\xi^{-\alpha}-\eta^{-\alpha}}{\alpha}
$$

i.e.

$$
h^{\prime}(r) \geq-\frac{r}{N \alpha}\left(\xi^{-\alpha}-\eta^{-\alpha}\right) .
$$

Integrating again from 0 to $r_{1}$, we have

$$
h\left(r_{1}\right)-h(0) \geq-\frac{r_{1}^{2}}{2 N \alpha}\left(\xi^{-\alpha}-\eta^{-\alpha}\right),
$$

hence

$$
r_{1}(\eta) \geq \sqrt{\frac{2 N \alpha\left(h(0)-h\left(r_{1}\right)\right)}{\xi^{-\alpha}-\eta^{-\alpha}}} \geq \sqrt{\frac{2 N \alpha(\eta-\xi)}{\xi^{-\alpha}-\eta^{-\alpha}}} .
$$

The bound when $\eta<\xi$ can be proved similarly. 


\section{Rupture Solutions}

In this section, we will consider radial solutions to (2.1) which are not smooth and prove Theorem 1.4 From Corollary 2.2] we need to consider $h \in C^{0}([0, \infty))$ such that $h(0)=0$ and $h$ satisfies (2.2) in $(0, \infty)$.

First, we check the growth rate of $h$ near the origin.

Lemma 4.1. Let $h$ be a radially symmetric rupture solution, then for any $\delta>0$, there exists positive constant $c_{1}$ such that

$$
h(r) \geq c_{1} r^{\frac{2}{\alpha+1}}
$$

holds for any $r \in[0, \delta]$.

Proof. Since $h$ is positive and smooth away from the origin, we only need to prove the bound for small $\delta$. Let $\delta>0$ be sufficiently small so that

$$
\frac{1}{\alpha} h^{-\alpha}(r)-p \geq \frac{1}{2 \alpha} h^{-\alpha}(r)
$$

holds for any $r \in(0, \delta]$. Now

$$
\left(r^{N-1} h^{\prime}\right)^{\prime}=r^{N-1}\left(\frac{h^{-\alpha}}{\alpha}-p\right) \geq \frac{1}{2 \alpha} h^{-\alpha} r^{N-1}
$$

implies $r^{N-1} h^{\prime}$ is monotone increasing in $(0, \delta]$. Since $h(0)=0$ and $h(r)$ is positive away from the origin, there exists a sequence $r_{i} \rightarrow 0$ such that $h^{\prime}\left(r_{i}\right)>0$. Hence, $h^{\prime}(r)>0$ for any $r \in(0, \delta]$. Integrating (4.1) from $\varepsilon$ to $r$, and using the fact that $h$ is increasing, we have

$$
r^{N-1} h^{\prime}(r)-\varepsilon^{N-1} h^{\prime}(\varepsilon) \geq \frac{1}{2 N \alpha} h^{-\alpha}(r)\left(r^{N}-\varepsilon^{N}\right) .
$$

Letting $\varepsilon \rightarrow 0$, we have

$$
r^{N-1} h^{\prime}(r) \geq \frac{1}{2 N \alpha} h^{-\alpha}(r) r^{N} .
$$

Hence for any $r \in(0, \delta]$,

$$
\frac{d}{d r} h^{\alpha+1}(r) \geq \frac{\alpha+1}{2 N \alpha} r .
$$

Integrating from 0 to $r$, we have

$$
h^{\alpha+1}(r) \geq \frac{\alpha+1}{4 N \alpha} r^{2},
$$

i.e. for any $r \in(0, \delta]$,

$$
h(r) \geq\left(\frac{\alpha+1}{4 N \alpha}\right)^{\frac{1}{\alpha+1}} r^{\frac{2}{\alpha+1}} .
$$

Lemma 4.2. Let $h$ be a radially symmetric rupture solution, then we have for some positive constant $c_{2}$,

$$
h(r) \leq c_{2} r^{\frac{2}{\alpha+1}}
$$

for any $r \in[0, \infty)$. 
Proof. Since $h$ is uniformly bounded at $\infty$, we only need to prove the inequality near the origin. First we claim

$$
\lim _{r \rightarrow 0^{+}} r^{N-1} h^{\prime}(r)=0 .
$$

From

$$
\left(r^{N-1} h^{\prime}\right)^{\prime}=r^{N-1}\left(\frac{h^{-\alpha}}{\alpha}-p\right),
$$

it follows that $r^{N-1} h^{\prime}$ is monotone increasing near the origin. Thus, if (4.2) is false, we would have

$$
r^{N-1} h^{\prime}(r) \geq c>0
$$

near the origin, hence

$$
h^{\prime}(r) \geq c r^{1-N}
$$

Since $r^{1-N}$ is not integrable near zero, the above inequality contradicts the fact that $h$ is continuous.

Given $\delta>0$, for any $r \in(0, \delta)$,

$$
\left(r^{N-1} h^{\prime}\right)^{\prime}=r^{N-1}\left(\frac{h^{-\alpha}}{\alpha}-p\right) \leq \frac{h^{-\alpha}}{\alpha} r^{N-1} \leq \frac{c_{1}^{-\alpha}}{\alpha} r^{N-1-\frac{2 \alpha}{\alpha+1}}
$$

by Lemma 4.1. Integrating from $\varepsilon$ to $r$, we obtain

$$
r^{N-1} h^{\prime}(r)-\varepsilon^{N-1} h^{\prime}(\varepsilon) \leq \frac{1}{\alpha\left(N-\frac{2 \alpha}{\alpha+1}\right)} c_{1}^{-\alpha}\left(r^{N-\frac{2 \alpha}{\alpha+1}}-\varepsilon^{N-\frac{2 \alpha}{\alpha+1}}\right)
$$

Letting $\varepsilon \rightarrow 0$, we have

$$
r^{N-1} h^{\prime}(r) \leq \frac{1}{\alpha\left(N-\frac{2 \alpha}{\alpha+1}\right)} c_{1}^{-\alpha} r^{N-\frac{2 \alpha}{\alpha+1}},
$$

i.e.,

$$
h^{\prime}(r) \leq \frac{1}{\alpha\left(N-\frac{2 \alpha}{\alpha+1}\right)} c_{1}^{-\alpha} r^{1-\frac{2 \alpha}{\alpha+1}} .
$$

Integrating from 0 to $r$, we have, for any $r \in(0, \delta)$,

$$
h(r) \leq \frac{\alpha+1}{2 \alpha\left(N-\frac{2 \alpha}{\alpha+1}\right)} c_{1}^{-\alpha} r^{\frac{2}{\alpha+1}} .
$$

Lemmas 4.1 and 4.2 imply that $h(r)$ is of order $r^{\frac{2}{\alpha+1}}$ near the origin. Now we write

$$
h=c^{*} \varphi(r) r^{\frac{2}{\alpha+1}},
$$

where

$$
c^{*}=\left[\frac{2 \alpha}{\alpha+1}\left(N-2+\frac{2}{\alpha+1}\right)\right]^{-\frac{1}{\alpha+1}} .
$$

Observe that $h=c^{*} r^{\frac{2}{\alpha+1}}$ is a solution of

$$
\triangle h-\frac{1}{\alpha} h^{-\alpha}=0
$$


in $(0, \infty)$. Direct calculation yields

$$
\varphi^{\prime \prime}+(A+1) \frac{\varphi^{\prime}}{r}+\frac{g(\varphi)}{r^{2}}+C r^{-\frac{2}{\alpha+1}}=0
$$

where

and

$$
A=N-2+\frac{4}{\alpha+1}>0, \quad C=\frac{p}{c^{*}}>0
$$

$$
g(\varphi)=\frac{2}{\alpha+1}\left(N-2+\frac{2}{\alpha+1}\right)\left(\varphi-\varphi^{-\alpha}\right) .
$$

Lemmas 4.1 and 4.2 imply that

$$
0<\liminf _{r \rightarrow 0^{+}} \varphi(r) \leq \limsup _{r \rightarrow 0^{+}} \varphi(r)<\infty .
$$

On the other hand, let $\varphi$ be a positive solution of (4.4) satisfying (4.5). Then $h$ defined by (4.3) is a rupture solution.

Locally, there exists at least one solution of (4.4) with initial values

$$
\varphi(0)=1, \varphi^{\prime}(0)=0 .
$$

To see this, we rewrite the equation as

$$
\varphi^{\prime \prime}+(A+1) \frac{\varphi^{\prime}}{r}+\frac{g^{\prime}(1)(\varphi-1)}{r^{2}}+\frac{g(\varphi)-g^{\prime}(1)(\varphi-1)}{r^{2}}+C r^{-\frac{2}{\alpha+1}}=0
$$

Denoting

$$
\psi=\varphi-1,
$$

we have

$$
\psi^{\prime \prime}+(A+1) \frac{\psi^{\prime}}{r}+\frac{g^{\prime}(1) \psi}{r^{2}}+\frac{\tilde{g}(\psi)}{r^{2}}+C r^{-\frac{2}{\alpha+1}}=0
$$

where

$$
\tilde{g}(\psi)=g(\psi+1)-g^{\prime}(1) \psi
$$

satisfies $\tilde{g}(0)=0, \tilde{g}^{\prime}(0)=0$. Now let $a_{1}, a_{2}$ be two numbers satisfying

$$
a_{1}+a_{2}=A=N-2+\frac{4}{\alpha+1}, \quad a_{1} a_{2}=g^{\prime}(1)=2\left(N-2+\frac{2}{\alpha+1}\right),
$$

then the real parts of $a_{1}, a_{2}$ are both positive and it is easy to verify that

$$
\psi^{\prime \prime}+(A+1) \frac{\psi^{\prime}}{r}+\frac{g^{\prime}(1) \psi}{r^{2}}=r^{-a_{2}-1}\left(r^{a_{2}-a_{1}+1}\left(r^{a_{1}} \psi\right)_{r}\right)_{r} .
$$

Hence, we have

$$
\begin{aligned}
\psi & =-r^{-a_{1}} \int_{0}^{r}\left\{s^{a_{1}-a_{2}-1} \int_{0}^{s} t^{a_{2}+1}\left(\frac{\tilde{g}(\psi(t))}{t^{2}}+C t^{-\frac{2}{\alpha+1}}\right) d t\right\} d s \\
& =-\frac{C}{\left(a_{1}+\frac{2 \alpha}{\alpha+1}\right)\left(a_{2}+\frac{2 \alpha}{\alpha+1}\right)} r^{\frac{2 \alpha}{\alpha+1}}-r^{-a_{1}} \int_{0}^{r}\left\{s^{a_{1}-a_{2}-1} \int_{0}^{s} t^{a_{2}-1} \tilde{g}(\psi(t)) d t\right\} d s .
\end{aligned}
$$

Let

$L \psi=-\frac{C}{\left(a_{1}+\frac{2 \alpha}{\alpha+1}\right)\left(a_{2}+\frac{2 \alpha}{\alpha+1}\right)} r^{\frac{2 \alpha}{\alpha+1}}-r^{-a_{1}} \int_{0}^{r}\left\{s^{a_{1}-a_{2}-1} \int_{0}^{s} t^{a_{2}-1} \tilde{g}(\psi(t)) d t\right\} d s$,

then for $\delta$ sufficiently small, $L$ is a contraction mapping from

$$
X=\{\psi \in C([0, \delta]):|\psi(r)| \leq \delta \text { for any } r \in[0, \delta]\}
$$


into itself. Here $L$ is a real mapping even though $a_{1}, a_{2}$ could be complex numbers. Let $\psi$ be the unique fixed point of $L$ in $X$, then $\varphi=1+\psi$ is a solution to (4.4) satisfying (4.6).

Let $\varphi$ be the local solution of (4.4) we just constructed, then $h$ defined by (4.3) is continuous with $h(0)=0$ and satisfies (2.2) in $(0, \delta)$. Such solution can be uniquely extended to a solution in $(0, \infty)$ which converges to $\xi$ by Lemma 2.8 Thus we have constructed a global rupture solution.

Remark 4.3. From the bounds in Lemmas 4.1 and 4.2. it is easy to see that the rupture solution we constructed is actually a weak solution of (2.1) in $\mathbb{R}^{N}, N \geq 2$. More precisely, we have

$$
h \in W_{\text {loc }}^{2, P}\left(\mathbb{R}^{N}\right), h^{-\alpha} \in L_{\text {loc }}^{P}\left(\mathbb{R}^{N}\right) \text { for any } 1 \leq P<\frac{\alpha+1}{2 \alpha} N,
$$

and

$$
\int_{\mathbb{R}^{N}} h \triangle \phi=\int_{\mathbb{R}^{N}}\left(\frac{1}{\alpha} h^{-\alpha}-p\right) \phi
$$

holds for any $\phi \in C_{0}^{\infty}\left(\mathbb{R}^{N}\right)$.

From the proof of Lemma 4.1 we have $h^{\prime}>0$ near the origin, so we can define

$$
r_{1}=\min \left\{r>0: h^{\prime}(r)=0\right\},
$$

the existence of $r_{1}$ is guaranteed by Lemma 2.4 Furthermore, as in the smooth solution case, we have a sequence $\left\{r_{k}\right\}_{k=1}^{\infty}$ such that for each $k, h$ is a rupture solution of

$$
\left\{\begin{array}{lll}
\triangle h=\frac{1}{\alpha} h^{-\alpha}-p & \text { in } & B_{r_{k}}(0), \\
\frac{\partial h}{\partial \nu}=0 & \text { on } & \partial B_{r_{k}}(0) .
\end{array}\right.
$$

In the remaining part of this section, we will show that the rupture solution to (2.2) is actually unique.

In (4.4), with

direct calculation yields

$$
r=e^{-t}, \quad \phi(t)=\varphi(r),
$$

$$
\phi_{t t}-A \phi_{t}+g(\phi)+C e^{-\frac{2 \alpha}{\alpha+1} t}=0
$$

on $(-\infty, \infty)$.

Lemma 4.4. There exists a unique global solution to (4.7) satisfying

$$
0<\liminf _{t \rightarrow \infty} \phi(t) \leq \limsup _{t \rightarrow \infty} \phi(t)<\infty .
$$

Noticing that $r \rightarrow 0^{+}$is equivalent to $t \rightarrow \infty$, the uniqueness of rupture solution follows from Lemma 4.4 Before proving Lemma 4.4 we first study the behavior of $\phi$ at $\infty$.

We write

$$
G(\phi)=\frac{2}{\alpha+1}\left(N-2+\frac{2}{\alpha+1}\right)\left(\frac{\phi^{2}}{2}+\frac{\phi^{1-\alpha}}{\alpha-1}\right),
$$

hence,

$$
G^{\prime}(\phi)=g(\phi) .
$$

Multiplying equation (4.7) with $\phi_{t}$, we have

$$
\frac{d}{d t}\left(\frac{\phi_{t}^{2}}{2}+G(\phi)\right)=A \phi_{t}^{2}-C e^{-\frac{2 \alpha}{\alpha+1} t} \phi_{t} \geq \frac{A}{2} \phi_{t}^{2}-\frac{C^{2}}{2 A} e^{-\frac{4 \alpha}{\alpha+1} t} .
$$


Lemma 4.5. Let $\phi$ be a global solution to (4.7) satisfying [4.8), then

$$
-\infty<\liminf _{t \rightarrow \infty} \phi_{t}(t) \leq \limsup _{t \rightarrow \infty} \phi_{t}(t)<\infty
$$

and

$$
\int_{0}^{\infty} \phi_{t}^{2}<\infty
$$

Furthermore, the limit

$$
\lim _{t \rightarrow \infty}\left(\frac{\phi_{t}^{2}}{2}+G(\phi)\right)
$$

exists and is finite.

Proof. If $\phi_{t}$ is unbounded at $\infty$, then $\frac{\phi_{t}^{2}}{2}+G(\phi)$ will be unbounded, so there exists a sequence $\left\{t_{k}\right\}_{k=1}^{\infty}$ with $\lim _{k \rightarrow \infty} t_{k}=\infty$, such that

$$
\lim _{k \rightarrow \infty}\left(\frac{\phi_{t}^{2}\left(t_{k}\right)}{2}+G\left(\phi\left(t_{k}\right)\right)\right)=\infty .
$$

For any $t>t_{k}$, integrating

$$
\frac{d}{d t}\left(\frac{\phi_{t}^{2}}{2}+G(\phi)\right) \geq-\frac{C^{2}}{2 A} e^{-\frac{4 \alpha}{\alpha+1} t}
$$

from $t_{k}$ to $t$, we have

$$
\begin{aligned}
& \frac{\phi_{t}^{2}(t)}{2}+G(\phi(t)) \geq \frac{\phi_{t}^{2}\left(t_{k}\right)}{2}+G\left(\phi\left(t_{k}\right)\right)-\int_{t_{k}}^{t} \frac{C^{2}}{2 A} e^{-\frac{4 \alpha}{\alpha+1} s} d s \\
\geq & \frac{\phi_{t}^{2}\left(t_{k}\right)}{2}+G\left(\phi\left(t_{k}\right)\right)-\frac{(\alpha+1) C^{2}}{8 \alpha A} .
\end{aligned}
$$

Hence,

$$
\lim _{t \rightarrow \infty}\left(\frac{\phi_{t}^{2}}{2}+G(\phi)\right)=\infty
$$

From (4.8), $G(\phi)$ is bounded at $\infty$, so we deduce

$$
\lim _{t \rightarrow \infty} \frac{\phi_{t}^{2}}{2}=\infty
$$

which is impossible for bounded $\phi$. The $L^{2}(0, \infty)$ bound of $\phi_{t}$ follows from (4.9) and the fact that $\frac{\phi_{t}^{2}}{2}+G(\phi)$ is bounded at $\infty$. Finally, since the right hand side of

$$
\frac{d}{d t}\left(\frac{\phi_{t}^{2}}{2}+G(\phi)\right)=A \phi_{t}^{2}-C e^{-\frac{2 \alpha}{\alpha+1} t} \phi_{t}
$$

is absolutely integrable at $\infty$, we have for any $t_{0}$,

$$
\lim _{t \rightarrow \infty}\left[\frac{\phi_{t}^{2}}{2}+G(\phi)\right]=\frac{\phi_{t}^{2}\left(t_{0}\right)}{2}+G\left(\phi\left(t_{0}\right)\right)+\int_{t_{0}}^{\infty}\left(A \phi_{t}^{2}-C e^{-\frac{2 \alpha}{\alpha+1} t} \phi_{t}\right)
$$

which is finite.

Lemma 4.6. If

$$
\lim _{t \rightarrow \infty} \phi=\varsigma,
$$

then

$$
\varsigma=1 .
$$


Proof. If $\varsigma \neq 1$, we first assume $\varsigma<1$, then for some small $\delta>0$,

$$
\phi_{t t}-A \phi_{t}=-g(\phi)-C e^{-\frac{2 \alpha}{\alpha+1} t}>\delta,
$$

for any $t \geq T_{0}$, where $T_{0}$ is a sufficiently large constant. Hence

$$
\left(e^{-A t} \phi_{t}\right)_{t}>\delta e^{-A t}
$$

for any $t \geq T_{0}$. Now since $\phi_{t}^{2}$ is integrable, we can choose $T_{1}>T_{0}$ with $\left|\phi_{t}\left(T_{1}\right)\right|$ sufficiently small. For any $t>T_{1}$, integrating (4.10) from $T_{1}$ to $t$, we have

$$
e^{-A t} \phi_{t}(t)-e^{-A T_{1}} \phi_{t}\left(T_{1}\right)>\frac{\delta}{A}\left(e^{-A T_{1}}-e^{-A t}\right) .
$$

Hence

$$
\phi_{t}(t)>\left[\frac{\delta}{A}\left(e^{-A T_{1}}-e^{-A t}\right)+e^{-A T_{1}} \phi_{t}\left(T_{1}\right)\right] e^{A t}>\frac{\delta}{2 A} e^{-A T_{1}} e^{A t}
$$

when $t$ is sufficiently large, which contradicts the boundedness of $\phi_{t}$ at $\infty$. The case $\varsigma>1$ can be treated in the same manner.

\section{Lemma 4.7.}

$$
\lim _{t \rightarrow \infty} G(\phi(t))=G(1)
$$

and hence

$$
\lim _{t \rightarrow \infty} \phi(t)=1 .
$$

Proof. Since $G(1)=\min G(\phi)$, if

$$
\lim _{t \rightarrow \infty}\left(\frac{\phi_{t}^{2}}{2}+G(\phi)\right)=G(1),
$$

then $\lim _{t \rightarrow \infty} G(\phi)$ exists and equals $G(1)$, as desired. We proceed by contradiction and assume that

$$
\lim _{t \rightarrow \infty}\left(\frac{\phi_{t}^{2}}{2}+G(\phi)\right)=L>G(1)
$$

We claim

$$
\liminf _{t \rightarrow \infty} G(\phi)<L .
$$

Otherwise,

$$
\lim _{t \rightarrow \infty} G(\phi)=L,
$$

which implies

$$
\lim _{t \rightarrow \infty} \phi=\varsigma
$$

for some $\varsigma$ with $G(\varsigma)=L$, a contradiction to Lemma 4.6 Hence, there exists a sequence $\left\{t_{k}\right\}_{k=1}^{\infty}$ such that $t_{k} \rightarrow \infty$ and

$$
G(1) \leq G\left(\phi\left(t_{k}\right)\right)<L-\delta
$$

for some $\delta>0$. Now we consider

$$
s_{k}=\sup \left\{s>t_{k}: \text { For any } t \in\left(t_{k}, s\right), G(\phi(t))<L-\frac{\delta}{2}\right\} .
$$

Observe that $s_{k}$ is finite. Otherwise $\phi_{t}^{2}>\frac{\delta}{8}$ for any $t$ sufficiently large, and then $\phi$ is monotone with derivative bounded away from zero, hence it will be unbounded, which gives a contradiction. Since $G\left(\phi\left(s_{k}\right)\right)=L-\frac{\delta}{2}$, we must have

$$
\left|\phi\left(t_{k}\right)-\phi\left(s_{k}\right)\right|>\delta_{1},
$$


where $\delta_{1}>0$ is a constant depending on $\delta, L$ and $G$. Since $\phi_{t}$ is bounded, we have

$$
\left|s_{k}-t_{k}\right|>\delta_{2} \equiv \frac{\delta_{1}}{\left\|\phi_{t}\right\|_{L^{\infty}}}>0
$$

However, for $t_{k}$ so large that

$$
\frac{\phi_{t}^{2}}{2}+G(\phi)>L-\frac{\delta}{4}
$$

on $\left(t_{k}, \infty\right)$, we have

$$
\phi_{t}^{2}(t)>\frac{\delta}{2}
$$

on $\left(t_{k}, s_{k}\right)$. Hence

$$
\int_{t_{k}}^{s_{k}} \phi_{t}^{2}>\frac{\delta}{2} \delta_{2}
$$

On the other hand, Lemma 4.5 says

$$
\lim _{k \rightarrow \infty} \int_{t_{k}}^{\infty} \phi_{t}^{2}=0
$$

which is a contradiction.

Now we are ready to prove Lemma 4.4

Proof of Lemma 4.4. Let $\phi$ and $\tilde{\phi}$ be two global solutions of (4.7) satisfying (4.8). Letting $\psi=\phi-\phi$, we have

$$
\psi_{t t}-A \psi_{t}+B(t) \psi=0
$$

Here

$$
A=N-2+\frac{4}{\alpha+1},
$$

and

$$
B(t)=\frac{2}{\alpha+1}\left(N-2+\frac{2}{\alpha+1}\right)\left(1+\frac{\left(\tilde{\phi}^{-\alpha}-\phi^{-\alpha}\right)}{\phi-\tilde{\phi}}\right) .
$$

Since

$$
\lim _{t \rightarrow \infty} \tilde{\phi}(t)=\lim _{t \rightarrow \infty} \phi(t)=1,
$$

we have

$$
\lim _{t \rightarrow \infty} B(t)=B_{0}=2\left(N-2+\frac{2}{\alpha+1}\right)>0 .
$$

It is easy to check that for any $\lambda$ such that

$$
\lambda^{2}-A \lambda+B_{0}=0,
$$

we have $\operatorname{Re} \lambda>0$. Since $\psi$ is bounded at $\infty$, Lemma 4.8 below with $\lambda_{0}=0$ implies $\psi \equiv 0$.

The following result seems standard and should be well-known. A proof is included here for the convenience of the reader. 
Lemma 4.8. Let $u$ satisfy the linear equation

$$
u_{t t}-A u_{t}+B(t) u=0 .
$$

Here $A$ is a constant, and $B(t)$ is a continuous function such that

$$
\lim _{t \rightarrow \infty} B(t)=B_{0} .
$$

Let $\lambda_{1}, \lambda_{2}$ be solutions of

$$
\lambda^{2}-A \lambda+B_{0}=0
$$

Suppose that there exists a constant $\lambda_{0}$ satisfying

$$
\lambda_{0}<\lambda_{m}=\min \left(\operatorname{Re} \lambda_{1}, \operatorname{Re} \lambda_{2}\right)
$$

such that, for some positive constants $T$ and $c$,

$$
|u(t)| \leq c e^{\lambda_{0} t}
$$

holds for any $t \geq T$, then $u \equiv 0$.

Proof. Let $u$ be any function satisfying (4.11). For any $\lambda \in\left(\lambda_{0}, \lambda_{m}\right)$, let $v=e^{-\lambda t} u$. It is easy to check

$$
v_{t t}-(A-2 \lambda) v_{t}+\left(B(t)-A \lambda+\lambda^{2}\right) v=0 .
$$

Since $\lambda<\lambda_{m}$, we have

$$
A-2 \lambda=\operatorname{Re} \lambda_{1}+\operatorname{Re} \lambda_{2}-2 \lambda \geq 2 \lambda_{m}-2 \lambda>0
$$

and

$$
B_{0}-A \lambda+\lambda^{2}>0
$$

Multiplying (4.12) with $v_{t}$, we obtain

$$
\frac{d}{d t}\left(v_{t}^{2}+\left(B_{0}-A \lambda+\lambda^{2}\right) v^{2}\right)=2(A-2 \lambda) v_{t}^{2}+2\left(B_{0}-B(t)\right) v v_{t} .
$$

For any $\varepsilon_{1}>0$ since

$$
\lim _{t \rightarrow \infty} B(t)=B_{0}
$$

there exists $T_{1}>0$, such that

$$
\left|\left(B_{0}-B(t)\right) v v_{t}\right| \leq \varepsilon_{1}\left(v_{t}^{2}+\left(B_{0}-A \lambda+\lambda^{2}\right) v^{2}\right)
$$

holds for any $t \geq T_{1}$. Hence for any $t \geq T_{1}$, we have

$$
\frac{d}{d t}\left(v_{t}^{2}+\left(B_{0}-A \lambda+\lambda^{2}\right) v^{2}\right) \leq 2\left(A-2 \lambda+\varepsilon_{1}\right)\left(v_{t}^{2}+\left(B_{0}-A \lambda+\lambda^{2}\right) v^{2}\right) .
$$

Gronwall's inequality then implies that for any $t \geq T_{1}$,

$$
v_{t}^{2}+\left(B_{0}-A \lambda+\lambda^{2}\right) v^{2} \leq c_{\varepsilon_{1}} e^{2\left(A-2 \lambda+\varepsilon_{1}\right) t}
$$

where

$$
c_{\varepsilon_{1}}=\left[v_{t}^{2}\left(T_{1}\right)+\left(B_{0}-A \lambda+\lambda^{2}\right) v^{2}\left(T_{1}\right)\right] e^{-2\left(A-2 \lambda+\varepsilon_{1}\right) T_{1}} .
$$

Now let $u_{1}$ be the solution of (4.11) in the Lemma such that

$$
\left|u_{1}(t)\right| \leq c e^{\lambda_{0} t}
$$


holds for any $t \geq T$. Then for any $\lambda \in\left(\lambda_{0}, \lambda_{m}\right)$ and for any $\varepsilon \in(0, A-2 \lambda)$, $v_{1}=e^{-\lambda t} u_{1}$ satisfies

$$
\begin{aligned}
& \frac{d}{d t}\left(v_{1, t}^{2}+\left(B_{0}-A \lambda+\lambda^{2}\right) v_{1}^{2}\right) \\
= & 2(A-2 \lambda) v_{1, t}^{2}+2\left(B_{0}-B(t)\right) v_{1} v_{1, t} \\
\geq & 2(A-2 \lambda-\varepsilon) v_{1, t}^{2}-v_{1}^{2}
\end{aligned}
$$

for any $t \geq T_{\varepsilon}$ if we choose $T_{\varepsilon} \geq T$ sufficiently large. Since $A-2 \lambda-\varepsilon>0$ and

$$
\left|v_{1}(t)\right| \leq c e^{-\left(\lambda-\lambda_{0}\right) t}
$$

holds for any $t \geq T$, we have, by similar arguments as in the proof of Lemma 4.5

$$
\left|v_{1, t}(t)\right| \leq C_{1}
$$

holds for some positive constant $C_{1}$ and for any $t \geq T_{\varepsilon}$. Hence for any $\lambda \in\left(\lambda_{0}, \lambda_{m}\right)$,

$$
\left|u_{1, t}(t)\right|=\left|e^{\lambda t} v_{1, t}(t)+\lambda e^{\lambda t} v_{1}(t)\right| \leq C_{2} e^{\lambda t}
$$

holds for some positive constant $C_{2}$ and for any $t \geq T_{\varepsilon}$. Especially, for any $\varepsilon_{2} \in$ $\left(0, \lambda_{m}-\lambda_{0}\right)$, we have

$$
\left|u_{1, t}(t)\right| \leq C_{2} e^{\left(\lambda_{0}+\varepsilon_{2}\right) t}
$$

holds for any $t \geq T_{\varepsilon}$ where $C_{2}$ is a large constant. Now for fixed $\lambda \in\left(\lambda_{0}, \lambda_{m}\right)$, we have

$$
\left|v_{1, t}\right|=\left|e^{-\lambda t} u_{1, t}-\lambda e^{-\lambda t} u_{1}\right| \leq C_{3} e^{\left(\lambda_{0}-\lambda+\varepsilon_{2}\right) t}
$$

for any $t \geq T_{\varepsilon}$ where $C_{3}$ is a large constant. If $u_{1}$ is a nontrivial solution to (4.11), then $v_{1}$ is a nontrivial solution to (4.12). Let $v_{2}$ be another solution of (4.12) which is linearly independent of $v_{1}$. Then (4.13) holds for $v_{2}$. Combining with (4.14) and (4.15), we have for any $t \geq \max \left\{T_{1}, T_{\varepsilon}\right\}$,

$$
W(t)=v_{1} v_{2, t}-v_{2} v_{1, t}
$$

satisfies

$$
\begin{aligned}
& |W(t)| \leq\left(v_{1, t}^{2}+v_{1}^{2}\right)^{\frac{1}{2}}\left(v_{2, t}^{2}+v_{2}^{2}\right)^{\frac{1}{2}} \\
\leq & C_{4} e^{\left(\lambda_{0}-\lambda+\varepsilon_{2}\right) t} \cdot e^{\left(A-2 \lambda+\varepsilon_{1}\right) t}=C_{4} e^{\left(A-2 \lambda+\lambda_{0}-\lambda+\varepsilon_{1}+\varepsilon_{2}\right) t} .
\end{aligned}
$$

On the other hand, since

$$
W^{\prime}(t)=(A-2 \lambda) W(t)
$$

we have

$$
W(t)=W(0) e^{(A-2 \lambda) t}
$$

Choosing $\varepsilon_{1}$ and $\varepsilon_{2}$ small enough so that

$$
\lambda_{0}-\lambda+\varepsilon_{1}+\varepsilon_{2}<0,
$$

we conclude from (4.16) and (4.17) that $W(t) \equiv 0$ which contradicts to the assumption that $v_{1}, v_{2}$ are two linearly independent solutions. Hence $v_{1} \equiv 0$ and $u_{1} \equiv 0$. 


\section{SCAling OF SOlutions}

In this section, we will use a scaling argument to prove Theorems 1.2 and 1.6

Let $h^{p, \eta}$ be the unique solution to (2.2) satisfying $h(0)=\eta \neq(\alpha p)^{-\frac{1}{\alpha}}$. When $\eta=0, h^{p, 0}$ is the unique rupture solution. Let $r_{k}^{p, \eta}, k=1,2, \cdots$, be the increasing sequence of positive critical points of $h^{p, \eta}$. Then

$$
h^{p, \eta, k}(x)=\left(r_{k}^{p, \eta}\right)^{-\frac{2}{1+\alpha}} h^{p, \eta}\left(r_{k}^{p, \eta}|x|\right)
$$

satisfies

$$
\left\{\begin{array}{lll}
\triangle h=\frac{1}{\alpha} h^{-\alpha}-p^{p, \eta, k} & \text { in } & B_{1}(0) \\
\frac{\partial h}{\partial \nu}=0 & \text { on } & \partial B_{1}(0)
\end{array}\right.
$$

with

$$
p^{p, \eta, k}=p\left(r_{k}^{p, \eta}\right)^{\frac{2 \alpha}{1+\alpha}}
$$

Let

$$
\bar{h}(p, \eta, k)=\frac{1}{\left|B_{1}(0)\right|} \int_{B_{1}(0)} h^{p, \eta, k}(x) d x=\frac{\left(r_{k}^{p, \eta}\right)^{-\frac{1}{2}}}{\left|B_{r_{k}}(0)\right|} \int_{B_{r_{k}}(0)} h^{p, \eta}(x) d x .
$$

Then $h^{p, \eta, k}(x)$ is a solution to (1.14) with

$$
\bar{h}=\bar{h}(p, \eta, k) \text {. }
$$

Lemma 5.1. For any $p>0$ and $\eta \geq 0, \eta \neq(\alpha p)^{-\frac{1}{\alpha}}$,

$$
h^{p, \eta}(x)=(\alpha p)^{-\frac{1}{\alpha}} h^{\frac{1}{\alpha},(\alpha p)^{\frac{1}{\alpha}} \eta}\left((\alpha p)^{\frac{1+\alpha}{2 \alpha}} x\right) .
$$

Proof. Let

$$
f(x)=(\alpha p)^{-\frac{1}{\alpha}} h^{\frac{1}{\alpha},(\alpha p)^{\frac{1}{\alpha}} \eta}\left((\alpha p)^{\frac{1+\alpha}{2 \alpha}} x\right),
$$

we have $f(0)=\eta$ and

$$
\begin{aligned}
& \triangle f(x)=\alpha p\left(\triangle h^{\frac{1}{\alpha},(\alpha p)^{\frac{1}{\alpha}} \eta}\right)\left((\alpha p)^{\frac{1+\alpha}{2 \alpha}} x\right) \\
& =\alpha p\left(\frac{1}{\alpha}\left(h^{\frac{1}{\alpha},(\alpha p)^{\frac{1}{\alpha}} \eta}\right)^{-\alpha}-\frac{1}{\alpha}\right)=\frac{1}{\alpha} f^{-\alpha}-p .
\end{aligned}
$$

So the lemma follows from the uniqueness of the radial solution.

Corollary 5.2. For each $k$,

$$
r_{k}^{p, \eta}=(\alpha p)^{-\frac{1+\alpha}{2 \alpha}} r_{k}^{\frac{1}{\alpha},(\alpha p)^{\frac{1}{\alpha}} \eta}, \quad h^{p, \eta, k}=h^{\frac{1}{\alpha},(\alpha p)^{\frac{1}{\alpha}} \eta, k}, \quad p^{p, \eta, k}=p^{\frac{1}{\alpha},(\alpha p)^{\frac{1}{\alpha}} \eta, k}
$$

and

$$
\bar{h}(p, \eta, k)=\bar{h}\left(\frac{1}{\alpha},(\alpha p)^{\frac{1}{\alpha}} \eta, k\right) .
$$

Now we are ready to prove Theorem 1.2

Proof of Theorem [1.2. Lemma 2.5] implies that for fixed $\eta>1, r_{k}^{\frac{1}{\alpha}, \eta}, k=1,2, \cdots$, are well seperated. Hence, we can apply standard ODE theory to conclude that for each $k=1,2, \cdots, r_{k}^{\frac{1}{\alpha}, \eta}$, viewed as a function of $\eta$, is continuous in $(1, \infty)$. 
From Lemma 3.2 and noticing $r_{k}^{\frac{1}{\alpha}, \eta}$ is monotone increasing in $k$, we have for each $k=1,2, \cdots$,

$$
\lim _{\eta \rightarrow \infty} r_{k}^{\frac{1}{\alpha}, \eta}=\infty
$$

Let

$$
R_{k}=\inf _{\eta>1} r_{k}^{\frac{1}{\alpha}, \eta}
$$

then $R_{k}$ is monotone nondecreasing in $k$. Furthermore, the interval $\left(R_{k}, \infty\right)$ is contained in the range of $r_{k}^{\frac{1}{\alpha}, \eta}$. Given $R>0$, let

$$
p_{k}=\frac{1}{\alpha}\left(\frac{R_{k}}{R}\right)^{\frac{2 \alpha}{1+\alpha}}
$$

then $p_{k}$ is monotone nondecreasing in $k$. For any $p>p_{k}$, and for any $1 \leq i \leq k$, let $\eta_{i} \in\left(R_{i}, \infty\right)$ be such that

$$
p=\frac{1}{\alpha}\left(\frac{r_{i}^{\frac{1}{\alpha}, \eta_{i}}}{R}\right)^{\frac{2 \alpha}{1+\alpha}}
$$

Then we have

$$
r_{i}^{p,(\alpha p)^{-\frac{1}{\alpha}} \eta_{i}}=(\alpha p)^{-\frac{1+\alpha}{2 \alpha}} r_{i}^{\frac{1}{\alpha}, \eta_{i}}=R,
$$

i.e., $h^{p,(\alpha p)^{-\frac{1}{\alpha}} \eta_{i}}$ is a nontrivial smooth radial solution to (1.8) in $B_{R}(0)$. Since $h^{p,(\alpha p)^{-\frac{1}{\alpha}} \eta_{i}}$, viewed as a function in $r$, has exactly $i-1$ critical points in $(0, R)$, we have found $k$ distinctive solutions as desired.

Remark 5.3. For fixed $k \geq 1$, numerical computation suggests that $r_{k}^{\frac{1}{\alpha}, \eta}$ is not monotone increasing for $\eta \in[0,1) \cup(1, \infty)$. Hence, given $p>p_{0}$, we may have two different solutions with the same number of critical points in $(0, R)$.

From Corollary 5.2 to get a solution to (1.14) through scaling, we can fix either $p$ or $\eta$. Without loss of generality, we assume $p=\frac{1}{\alpha}$, and $\eta \neq 1, \eta \geq 0$.

Proof of Theorem [1.6. All radial solutions can be obtained by scaling. So (1.14) has a rupture solution only when

$$
\bar{h}=\bar{h}_{k} \equiv \bar{h}\left(\frac{1}{\alpha}, 0, k\right) .
$$

Let

then

$$
r_{k} \equiv r_{k}^{\frac{1}{\alpha}, 0}
$$

$$
\bar{h}_{k}=\frac{\left(r_{k}\right)^{-\frac{1}{2}}}{\left|B_{r_{k}}(0)\right|} \int_{B_{r_{k}}(0)} h^{\frac{1}{\alpha}, 0}(x) d x .
$$

Since

$$
\lim _{r \rightarrow \infty} h^{\frac{1}{\alpha}, 0}(r)=1,
$$

we have

$$
\lim _{k \rightarrow \infty} \frac{1}{\left|B_{r_{k}}(0)\right|} \int_{B_{r_{k}}(0)} h^{\frac{1}{\alpha}, 0}(x) d x=1 .
$$

Hence the conclusion that

$$
\lim _{k \rightarrow \infty} \sqrt{k \pi} \bar{h}_{k}=1
$$

follows from Corollary 2.9 


\section{ACKNOWLEDGEMENTS}

We wish to thank Richard Laugesen, Mary Pugh and Dejan Slepčev and two anonymous referees for helpful comments. In particular, we wish to thank Mary Pugh for pointing out a related work of A. L. Bertozzi, G. Grün and T.P. Witelski [5. The research is supported in part by the NSF.

\section{REFERENCES}

[1] Robert Almgren, Andrea Bertozzi, and Michael P. Brenner. Stable and unstable singularities in the unforced Hele-Shaw cell. Phys. Fluids, 8(6):1356-1370, 1996.

[2] G. I. Barenblatt, E. Beretta, and M. Bertsch. The problem of the spreading of a liquid film along a solid surface: a new mathematical formulation. Proc. Nat. Acad. Sci. U.S.A., 94(19):10024-10030, 1997.

[3] Elena Beretta. Selfsimilar source solutions of a fourth order degenerate parabolic equation. Nonlinear Anal., 29(7):741-760, 1997.

[4] Andrew J. Bernoff and Andrea L. Bertozzi. Singularities in a modified Kuramoto-Sivashinsky equation describing interface motion for phase transition. Phys. D, 85(3):375-404, 1995.

[5] A. L. Bertozzi, G. Grün, and T. P. Witelski. Dewetting films: bifurcations and concentrations. Nonlinearity, 14(6):1569-1592, 2001.

[6] A. L. Bertozzi and M. Pugh. The lubrication approximation for thin viscous films: regularity and long-time behavior of weak solutions. Comm. Pure Appl. Math., 49(2):85-123, 1996.

[7] A. L. Bertozzi and M. C. Pugh. Finite-time blow-up of solutions of some long-wave unstable thin film equations. Indiana Univ. Math. J., 49(4):1323-1366, 2000.

[8] Andrea L. Bertozzi. The mathematics of moving contact lines in thin liquid films. Notices Amer. Math. Soc., 45(6):689-697, 1998.

[9] Andrea L. Bertozzi, Michael P. Brenner, Todd F. Dupont, and Leo P. Kadanoff. Singularities and similarities in interface flows. In Trends and perspectives in applied mathematics, volume 100 of Appl. Math. Sci., pages 155-208. Springer, New York, 1994.

[10] Michiel Bertsch, Roberta Dal Passo, Harald Garcke, and Günther Grün. The thin viscous flow equation in higher space dimensions. Adv. Differential Equations, 3(3):417-440, 1998.

[11] Peter Constantin, Todd F. Dupont, Raymond E. Goldstein, Leo P. Kadanoff, Michael J. Shelley, and Su-Min Zhou. Droplet breakup in a model of the Hele-Shaw cell. Phys. Rev. E (3), 47(6):4169-4181, 1993.

[12] Roberta Dal Passo, Harald Garcke, and Günther Grün. On a fourth-order degenerate parabolic equation: global entropy estimates, existence, and qualitative behavior of solutions. SIAM J. Math. Anal., 29(2):321-342 (electronic), 1998.

[13] Manuel A. del Pino and Gaston E. Hernandez. Solvability of the Neumann problem in a ball for $-\Delta u+u^{-v}=h(|x|), v>1$. J. Differential Equations, 124(1):108-131, 1996.

[14] Todd F. Dupont, Raymond E. Goldstein, Leo P. Kadanoff, and Su-Min Zhou. Finite-time singularity formation in Hele-Shaw systems. Phys. Rev. E (3), 47(6):4182-4196, 1993.

[15] P. Ehrhard. The spreading of hanging drops. J. Colloid \& Interface, 168(1):242-246, 19994.

[16] Günther Grün. Droplet spreading under weak slippage - existence for the Cauchy problem. Comm. Partial Differential Equations, 29(11-12):1697-1744, 2004.

[17] Philip Hartman. Ordinary differential equations. John Wiley \& Sons Inc., New York, 1964.

[18] Huiqiang Jiang and Fanghua Lin. Zero set of Sobolev functions with negative power of integrability. Chinese Ann. Math. Ser. B, 25(1):65-72, 2004.

[19] R. S. Laugesen and M. C. Pugh. Linear stability of steady states for thin film and CahnHilliard type equations. Arch. Ration. Mech. Anal., 154(1):3-51, 2000.

[20] R. S. Laugesen and M. C. Pugh. Properties of steady states for thin film equations. European J. Appl. Math., 11(3):293-351, 2000.

[21] R. S. Laugesen and M. C. Pugh. Energy levels of steady states for thin-film-type equations. J. Differential Equations, 182(2):377-415, 2002.

[22] Richard S. Laugesen and Mary C. Pugh. Heteroclinic orbits, mobility parameters and stability for thin film type equations. Electron. J. Differential Equations, pages No. 95, 29 pp. (electronic), 2002.

[23] T. G. Myers. Thin films with high surface tension. SIAM Rev., 40(3):441-462 (electronic), 1998. 
[24] A. Oron, S. H. Davis, and S. G. Bankoff. Nonlinear theory of film rupture. Rev. Mod. Phys., 69(3):931-980, 1997.

[25] Michael J. Shelley, Raymond E. Goldstein, and Adriana I. Pesci. Topological transitions in Hele-Shaw flow. In Singularities in fluids, plasmas and optics (Heraklion, 1992), volume 404 of NATO Adv. Sci. Inst. Ser. C Math. Phys. Sci., pages 167-188. Kluwer Acad. Publ., Dordrecht, 1993.

[26] D. Slepčev and M. C. Pugh. Selfsimilar blowup of unstable thin-film equations. Indiana Univ. Math. J., 54(6):1697-1738, 2005.

[27] Malcolm B. Williams and Stephen H. Davis. Nonlinear theory of film rupture. J. Colloid Interf. Sci., 90(1):220-228, 1982.

[28] Thomas P. Witelski and Andrew J. Bernoff. Stability of self-similar solutions for van der Waals driven thin film rupture. Phys. Fluids, 11(9):2443-2445, 1999.

[29] Thomas P. Witelski and Andrew J. Bernoff. Dynamics of three-dimensional thin film rupture. Phys. D, 147(1-2):155-176, 2000.

[30] Wendy W. Zhang and John R. Lister. Similarity solutions for van der Waals rupture of a thin film on a solid substrate. Phys. Fluids, 11(9):2454-2462, 1999.

School of Mathematics, University of Minnesota, 127 Vincent Hall, 206 Church St. S.E., Minneapolis, MN 55455

E-mail address: hqjiang@math.umn.edu

School of Mathematics, University of Minnesota, 127 Vincent Hall, 206 Church St. S.E., Minneapolis, MN 55455

E-mail address: ni@math.umn.edu 